\title{
The Effects of Different Types of Steroids on Clinical Outcomes in Neonates with Meconium Aspiration Syndrome: A Systematic Review, Meta-Analysis and GRADE Assessment
}

\author{
Nanthida Phattraprayoon ${ }^{1}$, Teerapat Ungtrakul ${ }^{1}$ (D) and Wimonchat Tangamornsuksan ${ }^{1,2, *}$ \\ 1 Faculty of Medicine and Public Health, HRH Princess Chulabhorn College of Medical Science, \\ Chulabhorn Royal Academy, Bangkok 10210, Thailand; nanthida.pha@cra.ac.th (N.P.); \\ teerapat.ung@cra.ac.th (T.U.) \\ 2 Department of Health Research Methods, Evidence and Impact, McMaster University, \\ Hamilton, ON L8S 4L8, Canada \\ * Correspondence: wimonchat.tan@cra.ac.th
}

\section{check for} updates

Citation: Phattraprayoon, N.; Ungtrakul, T.; Tangamornsuksan, W. The Effects of Different Types of Steroids on Clinical Outcomes in Neonates with Meconium Aspiration Syndrome: A Systematic Review, Meta-Analysis and GRADE Assessment. Medicina 2021, 57, 1281. https://doi.org/10.3390/ medicina57111281

Academic Editor: Ralph Fingerhut

Received: 12 October 2021

Accepted: 18 November 2021

Published: 21 November 2021

Publisher's Note: MDPI stays neutral with regard to jurisdictional claims in published maps and institutional affiliations.

Copyright: (c) 2021 by the authors Licensee MDPI, Basel, Switzerland. This article is an open access article distributed under the terms and conditions of the Creative Commons Attribution (CC BY) license (https:// creativecommons.org/licenses/by/ $4.0 /)$.

\begin{abstract}
Background and Objectives: Meconium aspiration syndrome (MAS) is a condition caused by the aspiration of meconium-stainted amniotic fluid into the lungs, resulting in pulmonary inflammation, neonatal morbidity, and mortality. It is important that these MAS infants receive appropriate care to avoid further complications. Steroids have an anti-inflammatory effect and may be effective in the management of MAS. The objective of the this study was to evaluate the effect of different steroids on clinical outcomes in infants with MAS. Materials and Methods: We systematically searched of PubMed/Medline, Scopus, Embase, Clinical Trials.gov, and Cochrane Library databases from inception to 24 January 2021 without language restriction. Only randomized controlled trials (RCTs) evaluating the effects of steroids in neonates with MAS were included. We calculated relative risks and weighted mean differences (MDs) with 95\% confidence intervals (CIs) using a random-effects model to determine the associations between MAS and steroids and GRADE approach was employed for quality of evidence. The main outcomes measures were duration of respiratory distress, oxygen requirement, hospitalization, need for mechanical ventilation, death, and adverse drug reactions. Results: Seven RCTs involving 397 patients were analyzed. Nebulized budesonide and intravenous (IV) methylprednisolone shortened the duration of respiratory distress (MD, - 2.46 days; $95 \% \mathrm{CI},-3.09$ to -1.83 and $\mathrm{MD},-3.30$ days; $95 \% \mathrm{CI},-4.07$ to -2.52 , respectively) (moderate certainty). There was a reduction in duration of oxygen requirement in nebulized budesonide use (MD, -2.40 days; $95 \% \mathrm{CI},-3.40$ to -1.40 ) (low certainty) and IV methylprednisolone use (MD, -3.30 days; $95 \%$ CI,-4.07 to -2.52 ) (moderate certainty). Nebulized budesonide shortened hospitalization stay (MD, -4.47 days; $95 \%$ CI -8.64 to -0.30 days) (low certainty) as IV methylprednisolone use (MD, -7.23 days; $95 \% \mathrm{CI},-8.19$ to -6.07 days) (moderate certainty). None of steroids benefits in death (low certainty). Conclusions: Certain types of steroids may benefit the respiratory aspect, but there was no decrease in mortality in MAS infants.
\end{abstract}

Keywords: different types of steroids; meconium aspiration syndrome; clinical outcomes; systematic review and meta-analysis

\section{Introduction}

Meconium aspiration syndrome (MAS) is caused by aspiration of meconium-containing amniotic fluid into the lungs. The complex chemical composition of meconium may be responsible for pulmonary inflammation, termed chemical pneumonitis, and a risk of surfactant inactivation [1]. The aspirated meconium may also cause mechanical obstruction of small airways. In neonates with partial airway occlusion, the over-expanded lungs will increase air leakage complications [2]. Around $5 \%$ to $20 \%$ of meconium-stained amniotic fluid (MSAF) infants develop MAS [3,4], with mortality rate of 5\% [5,6]. The incidence of 
MAS is declining in developed countries with advances in obstetric practices and perinatal care $[7,8]$, however, MAS remains a significant respiratory problem and cause of neonatal mortality in developing countries $[9,10]$. Management of MAS mainly involves supportive care to ensure appropriate oxygenation and ventilation, including stabilization of the systemic circulation, in an effort to prevent other serious conditions such as persistent pulmonary hypertension of the newborn (PPHN) [11]. The pathophysiology of MAS depends on the severity of inflammation, and hence suppressing inflammation may improve clinical outcomes in MAS infants. Steroids can down-regulate proinflammatory cytokine production in vitro [12-14]. Using steroids to suppress inflammation in infants with MAS may also be beneficial. However, there is a lack of sufficient evidence to assess the potential benefits and detriments of the use of steroids in MAS [15]. Therefore, we conducted a systematic review and meta-analysis of the efficacy of different types of steroids in MAS.

\section{Materials and Methods}

The study protocol was registered in PROSPERO (registration number CRD42020211334). This systematic review and meta-analysis was performed according to the Preferred Reporting Items for Systematic Reviews and Meta-Analyses (PRISMA).

The main outcomes were the duration of respiratory distress, oxygen requirement, hospitalization, need for mechanical ventilation, death, complications and adverse drug reactions (ADRs).

\subsection{Search Strategy and Selection Criteria}

We performed a comprehensive and systematic search of the PubMed/Medline (U.S. National Library of Medicine, Bethesda, MD, USA), Scopus, Embase, Clinical Trials.gov, and Cochrane Library databases from inception to 24 January 2021 using keywords, synonyms, and other terms related to MAS and steroids without language restriction. Only randomized controlled trials (RCTs) were included. Additional studies were identified via the reference lists of selected articles.

Two reviewers (N.P. and W.T.) separately and independently screened and selected studies using the eligibility criteria. Any disagreements were resolved by discussion with a third reviewer (T.U.).

\subsection{Data Extraction and Risk-of-Bias (Quality) Assessment}

Two reviewers (N.P. and W.T.) separately extracted data from the included studies, including the study design and methodology, eligibility and diagnostic criteria, patient demographics, data collection method, definition of outcomes and outcomes parameters, and number of events. Study investigators were contacted for any missing data, unreported data, and additional details.

Two reviewers (N.P. and W.T.) independently evaluated the quality of the included studies using the Revised Cochrane risk-of-bias tool for randomized trials (RoB 2) [16]. Any disagreements were resolved by discussion with a third reviewer (T.U.).

\subsection{Data Analyses}

We calculated relative risks (RRs) and weighted mean differences (MDs) with 95\% confidence intervals (CIs) using the DerSimonian and Laird method with a random-effects model to determine the associations between MAS and steroids in neonates with MAS for dichotomous and continuous outcomes, respectively [17]. We performed a separate analysis based on the type of steroids and assessed statistical heterogeneity via Q-statistic and $\mathrm{I}^{2}$ tests. $p$-values of $\leq 0.05$ indicated heterogeneity between studies [18]. $\mathrm{I}^{2}$ values of $25 \%, 50 \%$, and $75 \%$ denoted low, moderate, and high heterogeneity across studies, respectively [19]. If $\geq 10$ studies proved eligible in each outcome, publication bias was evaluated using a funnel plot [20]. All statistical analyses were performed using Stata version 16.0 (StataCorp LLC, College Station, TX, USA). 


\subsection{Quality of Evidence}

We used the Grading of Recommendations Assessment, Development and Evaluation (GRADE) approach to rate the quality of evidence for each outcome as high, moderate, low, or very low [21]. The assessment included judgments addressing the risk of bias [22], imprecision [23], inconsistency [24], indirectness [25], and publication bias [26]. If there were serious concerns in any of these domains, we rated down the quality of evidence.

\section{Results}

\subsection{Search Strategy and Selection Criteria}

In total, 636 citations were identified by the database search (Figure 1). After screening titles and abstracts, 18 full texts were screened. Eleven studies met our inclusion criteria, but the full text of Davey et al. [27] was not assessed. Finally, 10 studies [28-37] were included in our systematic review, 7 of which $[29-33,35,36]$ were included in the meta-analysis. No additional articles were retrieved from the reference lists of the included studies.

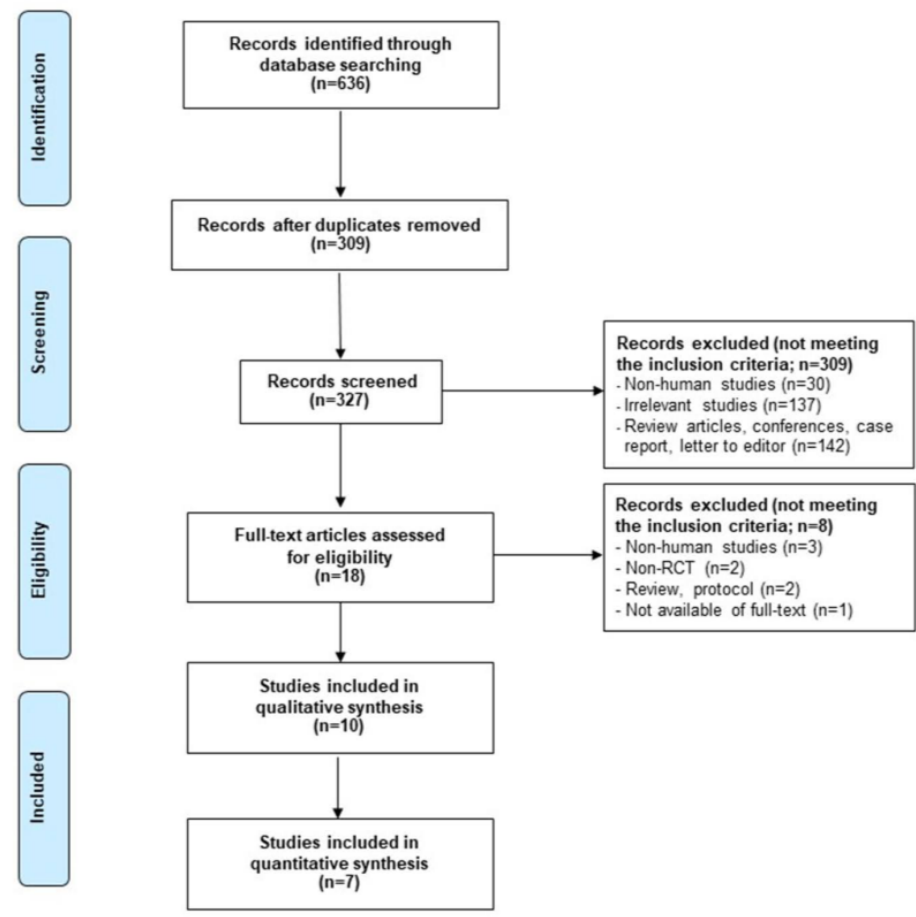

Figure 1. PRISMA flow diagram of study selection for the systematic review and meta-analysis.

3.2. Data Extraction and Risk-of-Bias Assessment Study Characteristics

The study characteristics and the maternal and infant characteristics are shown in Table 1, and Supplementary Table S1, respectively. Among the 10 studies, 1 compared lactose hydrous (placebo) and intravenous (IV) hydrocortisone [28]. Three studies compared IV normal saline solution (NSS) or no treatment versus IV dexamethasone [29,35,36]. Two studies compared nebulized NSS, IV 5\% dextrose, or no treatment (control) versus IV methylprednisolone or nebulized budesonide [30,31]. Two studies compared nebulized NSS versus nebulized budesonide [32,33]. One study assigned patients to receive either nebulized 3\% sodium chloride with IV NSS (placebo) or nebulized budesonide with IV methylprednisolone [37]. One study [34], patients received intratracheal instillation of porcine lung surfactant (PS) or intratracheal instillation of PS with budesonide. Definitions used in the included studies were as follows:

1. MAS $[28-34,36,37]$

- Delivery of MSAF infants and retrieval of meconium from below the larynx on endotracheal tube suction; 
- Development of respiratory distress within 4 to $6 \mathrm{~h}$ after birth and persistence beyond $24 \mathrm{~h}$;

- Chest X-ray findings of infiltrates, hyperinflation, and atelectasis;

- Absence of any other causes of respiratory distress.

2. Sepsis [30]

- $\quad$ Presence of clinical signs: poor feeding, weight loss, lethargy, temperature instability, sclerema, and capillary refill time of $>3$ s and;

- Positive blood culture, or;

- Two or more of the following laboratory abnormalities:

(a) Total leukocyte count of $<5000 / \mathrm{mm}^{3}$ or $>30,000 / \mathrm{mm}^{3}$;

(b) Immature/total neutrophil ratio of $>0.2$;

(c) Micro-erythrocyte sedimentation rate of $>5 \mathrm{~mm}$ in the first hour on the first day of life or $>15 \mathrm{~mm}$ at any time;

(d) Positive C-reactive protein.

\subsection{Risk-of-Bias Assessment}

The risk-of-bias assessment results are summarized in Supplementary Table S2. The allocation sequence was generated in 5 of 10 the studies [28,30-33], 1 of which used concealed allocation [30]. Three studies were double-blind RCTs [28,29,31] Loss to followup [28-37] and selective outcome reporting were adequate.

\subsection{Data Analyses}

The efficacy of steroids on clinical outcomes, ADRs, and complications in infants with MAS are shown in Figures 2 and 3, Table 2, and Supplementary Tables S3, S4 and Figure S1.

\subsubsection{Duration of Respiratory Distress}

Six RCTs provided evidence regarding respiratory distress [28,30-33,37]. With moderatequality evidence, nebulized budesonide appeared to reduce the duration of respiratory distress in 4 RCTs [30-33] (MD, -2.46 days; 95\% CI, -3.09 to -1.83 days) as well as IV methylprednisolone in 2 RCTs [30,31] (MD, -3.30 days; 95\% CI, -4.07 to -2.52 days) (Figure 2A, Table 2, and Supplementary Tables S3 and S4).

\subsubsection{Duration of Oxygen Requirement}

Eight RCTs [28-33,36,37] provided data regarding the duration of oxygen requirement. Both nebulized budesonide and IV methylprednisolone appeared to reduce the duration of oxygen requirement. Four RCTs [30-33] demonstrated this beneficial effect of nebulized budesonide with low-quality evidence (MD, -2.4 days; $95 \% \mathrm{CI},-3.4$ to -1.4 days). Two RCTs $[30,31]$ demonstrated this beneficial effect of IV methylprednisolone with moderate-quality evidence (MD, -3.30 days; $95 \% \mathrm{CI},-4.07$ to -2.52 days). Two RCTs [29,36] demonstrated the effect of dexamethasone, only one reported on the duration [29]. (Figure 2B, Table 2, and Supplementary Tables S3 and S4).

\subsubsection{Need for Mechanical Ventilation}

Six RCTs [28,30,33-36] determined the effect of steroids on the need for mechanical ventilation. Two studies $[35,36]$ showed no significant difference in the need for mechanical ventilation when using IV dexamethasone versus the control with very low-quality evidence (RR, 1.23; 95\% CI, 0.17 to 8.87) (Figure 2C, Table 2, and Supplementary Tables S3 and S4).

\subsubsection{Duration of Mechanical Ventilation}

Three RCTs [29,32,37] determined the effect of steroids on the duration of mechanical ventilation. Two RCTs [29,37] calculated the MD, which one RCT showed no difference in the duration of mechanical ventilation between dexamethasone and placebo (MD, -1.10 days; $95 \% \mathrm{CI},-2.79$ to 0.59 days) [29]. 
Table 1. Characteristics of included studies.

\begin{tabular}{|c|c|c|c|c|c|c|c|c|c|c|}
\hline Study & $\begin{array}{l}\text { Yeh et al., } 1977 \\
\text { [28] }\end{array}$ & $\begin{array}{l}\text { Wu et al., } 1999 \\
\text { [29] }\end{array}$ & $\begin{array}{l}\text { Basu et al., } 2007 \\
\text { [30] }\end{array}$ & $\begin{array}{l}\text { Tripathi et al., } \\
\text { 2007 [31] }\end{array}$ & $\begin{array}{l}\text { Suresh et al., } 2015 \\
\text { [32] }\end{array}$ & Garg et al., 2016 [33] & Tan et al., 2016 [34] & $\begin{array}{l}\text { Sangeetha et al., } \\
2017 \text { [35] }\end{array}$ & $\begin{array}{l}\text { Patil et al., } 2018 \\
\text { [36] }\end{array}$ & $\begin{array}{l}\text { Rana et al., } 2018 \\
\text { [37] }\end{array}$ \\
\hline Type of study & RCT & RCT & RCT & RCT & RCT & RCT & RCT & RCT & RCT & RCT \\
\hline Location & Illinois, USA & Taipei, Taiwan & Varanasi, India & New Delhi, India & Karnataka, India & Rajasthan, India & $\begin{array}{l}\text { Foshan Nanhai, } \\
\text { China }\end{array}$ & $\begin{array}{l}\text { Chidambaram, } \\
\text { India }\end{array}$ & Karnataka, India & $\begin{array}{l}\text { West Bengal, } \\
\text { India }\end{array}$ \\
\hline $\begin{array}{l}\text { Inclusion } \\
\text { criteria }\end{array}$ & $\begin{array}{ll}\text { - } & \text { Neonates } \\
& \text { with MAS }\end{array}$ & $\begin{array}{ll}- & \text { Neonates } \\
& \text { with MAS }\end{array}$ & $\begin{array}{l}\text { Neonates } \\
\text { with MAS }\end{array}$ & $\begin{array}{ll}- & \text { Full-term } \\
& \text { neonates } \\
- & \text { BW }>2000 \\
\text { - } & \text { MAS }\end{array}$ & $\begin{array}{ll}- & \text { Full-term } \\
& \text { neonates } \\
\text { - } & \text { MAS }\end{array}$ & $\begin{array}{ll}- & \text { Term neonates } \\
- & (\mathrm{GA} \geq 37 \\
& \text { weeks) } \\
- & \text { BW } \geq 2000 \mathrm{~g} \\
- & \text { Neonates with } \\
& \text { non-vigorous } \\
\text { meconium- } \\
\text { stained } \\
\text { amniotic fluid } \\
-\quad \text { Neonates with } \\
\text { MAS }\end{array}$ & $\begin{array}{l}\text { Neonates } \\
\text { with MAS }\end{array}$ & $\begin{array}{ll}- & \text { Term } \\
& \text { neonates } \\
& \text { with MAS } \\
- & \text { Admitted } \\
& \text { to NICU }\end{array}$ & $\begin{array}{ll}- & \text { GA } \geq 34 \\
& \text { weeks } \\
\text { - } & \text { Neonates } \\
& \text { with MAS }\end{array}$ & $\begin{array}{l}\text { Neonates } \\
\text { with MAS }\end{array}$ \\
\hline $\begin{array}{l}\text { Exclusion } \\
\text { criteria }\end{array}$ & - & - & $\begin{array}{ll}- & \text { Sepsis } \\
- & \text { Systemic } \\
- & \text { illness } \\
- & \text { Gross } \\
\text { congenital } \\
\text { malforma- } \\
\text { tions }\end{array}$ & $\begin{array}{ll}- & \text { Preterm } \\
- & \text { IUGR } \\
- & \text { Out-born } \\
& \text { babies } \\
- & \text { Congenital } \\
& \text { malforma- } \\
& \text { tion } \\
- & \begin{array}{l}\text { Denied } \\
\text { consent }\end{array}\end{array}$ & $\begin{array}{ll}- & \text { Sepsis } \\
- & \text { Systemic } \\
& \text { illness } \\
- & \text { Gross } \\
& \text { congenital } \\
& \text { malforma- } \\
& \text { tion } \\
- & \text { Preterm } \\
- & \text { IUGR } \\
- & \text { Denied } \\
\text { consent }\end{array}$ & $\begin{array}{ll}- & \text { Congenital } \\
\text { malformation } \\
\text { - } & \text { Grade 3 HIE } \\
- & \text { History } \\
\text { suggestive of } \\
\text { early-onset } \\
\text { sepsis }\end{array}$ & $\begin{array}{ll}- & \begin{array}{l}\text { Severe } \\
\text { congenital } \\
\text { malforma- } \\
\text { tions }\end{array} \\
- & \text { Serious } \\
\text { systemic } \\
\text { diseases } \\
-\quad & \text { Heart failure } \\
\text { - Renal } \\
\text { insufficiency } \\
\text { - Acute or } \\
\text { chronic } \\
\text { diseases }\end{array}$ & 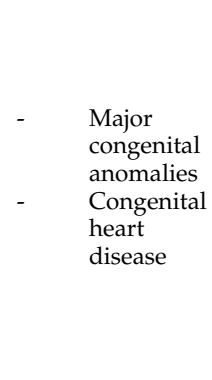 & $\begin{array}{ll}- & \begin{array}{l}\text { Congenital } \\
\text { malforma- } \\
\text { tion }\end{array} \\
\text { - } & \text { Denied } \\
\text { consent } \\
- & \text { Suspected } \\
\text { sepsis }\end{array}$ & $\begin{array}{ll}- & \text { Preterm } \\
- & \text { IUGR } \\
- & \text { Major } \\
& \text { congenital } \\
\text { malforma- } \\
\text { tions } \\
\text { - } & \text { Denied } \\
& \text { consent } \\
- & \text { Died } \\
\text { during } \\
\text { early } \\
\text { neonatal } \\
\text { period }\end{array}$ \\
\hline Randomization & $\begin{array}{l}\text { Random number } \\
\text { table }\end{array}$ & $\begin{array}{l}\text { Randomization } \\
\text { method not } \\
\text { mentioned }\end{array}$ & $\begin{array}{l}\text { Computer- } \\
\text { generated random } \\
\text { numbers }\end{array}$ & $\begin{array}{l}\text { Computer- } \\
\text { generated random } \\
\text { numbers }\end{array}$ & $\begin{array}{l}\text { Computer- } \\
\text { generated random } \\
\text { numbers }\end{array}$ & $\begin{array}{l}\text { Computer-generated } \\
\text { random numbers }\end{array}$ & $\begin{array}{l}\text { Randomization } \\
\text { method not } \\
\text { mentioned }\end{array}$ & $\begin{array}{l}\text { Randomization } \\
\text { method not } \\
\text { mentioned }\end{array}$ & $\begin{array}{l}\text { Randomization } \\
\text { method not } \\
\text { mentioned }\end{array}$ & $\begin{array}{l}\text { Randomization } \\
\text { method not } \\
\text { mentioned }\end{array}$ \\
\hline Study period & $\begin{array}{l}1 \text { year } \\
\text { (October 1974 to } \\
\text { September 1975) }\end{array}$ & NR & 1 year & 1 year & $\begin{array}{l}3 \text { months } \\
\text { (August to } \\
\text { October 2013) }\end{array}$ & $\begin{array}{l}5 \text { months } \\
\text { (May } 2010 \text { to December } \\
\text { 2010) }\end{array}$ & $\begin{array}{l}2 \text { years } \\
\text { (December 2013 to } \\
\text { December 2015) }\end{array}$ & 1 year & 18 months & $\begin{array}{l}3 \text { years } \\
\text { (April 2014 to } \\
\text { March 2017) }\end{array}$ \\
\hline
\end{tabular}


Table 1. Cont.

\begin{tabular}{|c|c|c|c|c|c|c|c|c|c|c|}
\hline Study & $\begin{array}{l}\text { Yeh et al., } 1977 \\
\text { [28] }\end{array}$ & $\begin{array}{l}\text { Wu et al., } 1999 \\
\text { [29] }\end{array}$ & $\begin{array}{l}\text { Basu et al., } 2007 \\
\text { [30] }\end{array}$ & $\begin{array}{l}\text { Tripathi et al., } \\
\text { 2007 [31] }\end{array}$ & $\begin{array}{l}\text { Suresh et al., } 2015 \\
\text { [32] }\end{array}$ & Garg et al., 2016 [33] & Tan et al., 2016 [34] & $\begin{array}{l}\text { Sangeetha et al., } \\
2017 \text { [35] }\end{array}$ & $\begin{array}{l}\text { Patil et al., } 2018 \\
\text { [36] }\end{array}$ & $\begin{array}{l}\text { Rana et al., } 2018 \\
\text { [37] }\end{array}$ \\
\hline $\begin{array}{l}\text { All groups } \\
\text { received }\end{array}$ & $\begin{array}{l}\text { Standard care as } \\
\text { protocol }\end{array}$ & $\begin{array}{l}\text { Standard care as } \\
\text { protocol }\end{array}$ & $\begin{array}{l}\text { Supportive } \\
\text { treatment as } \\
\text { protocol of } \\
\text { nursery }\end{array}$ & $\begin{array}{l}\text { Standard care as } \\
\text { unit protocol }\end{array}$ & $\begin{array}{l}\text { Supportive } \\
\text { treatment as } \\
\text { protocol of } \\
\text { nursery }\end{array}$ & $\begin{array}{l}\text { Standard care as } \\
\text { protocol }\end{array}$ & $\begin{array}{l}\text { PS } 100 \mathrm{mg} / \mathrm{kg} \\
\text { within } 2 \mathrm{~h}\end{array}$ & $\begin{array}{l}\text { Standard care as } \\
\text { unit protocol }\end{array}$ & $\begin{array}{l}\text { Standard care as } \\
\text { protocol }\end{array}$ & Standard care \\
\hline Control & $\begin{array}{l}\text { Placebo } \\
\text { (lactose hydrous) }\end{array}$ & NSS & None & $\begin{array}{ll}- & \text { Nebulized } \\
\text { NSS } \\
\text { - } \quad \text { \% } \\
\text { Dextrose } \\
\text { IV }\end{array}$ & Nebulized NSS & $\begin{array}{l}\text { - } \\
2.5 \mathrm{~mL} \text { within } \\
2 \mathrm{~h} \text { and at } 12 \mathrm{~h} \\
\text { after birth via } \\
\text { nebulizer } \\
\text { system }\end{array}$ & $\begin{array}{l}\text { - } \quad \text { PS } 100 \\
\mathrm{mg} / \mathrm{kg} \text { by } \\
\text { intratracheal } \\
\text { instillation } \\
\text { within } 2 \mathrm{~h} \text { of } \\
\text { birth }\end{array}$ & None & None & $\begin{array}{ll}- & \text { Nebulized } \\
& 3 \% \mathrm{NaCl} \\
- & \text { IV NSS }\end{array}$ \\
\hline $\begin{array}{l}\text { Comparator } \\
\text { (1) }\end{array}$ & $\begin{array}{l}\text { Hydrocortisone } \\
20 \mathrm{mg} / \mathrm{kg} \text { IV for } \\
\text { first bolus dose, } \\
\text { then continue q } \\
12 \mathrm{~h} \text { for } 4 \text { more } \\
\text { doses }\end{array}$ & $\begin{array}{l}\text { Dexamethas } \\
1 \mathrm{mg} / \mathrm{kg} \text { IV for } \\
\text { initial dose, then } \\
0.5 \mathrm{mg} / \mathrm{kg} \mathrm{q} 12 \mathrm{~h} \\
\text { for days } 1-3 \text {, then } \\
0.25 \mathrm{mg} / \mathrm{kg} \mathrm{q} \\
12 \mathrm{~h} \text { for days } 4-7 \\
\text { (started shortly } \\
\text { after birth) }\end{array}$ & $\begin{array}{l}\text { Methyl- } \\
\text { prednisolone } \\
0.5 \mathrm{mg} / \mathrm{kg} / \text { day IV } \\
\text { q } 12 \mathrm{~h} \text { for } 7 \text { days } \\
\text { (started } 24-36 \mathrm{~h} \\
\text { after birth) }\end{array}$ & $\begin{array}{ll}\text { - } & \text { Nebulized } \\
\text { NSS } \\
\text { Methyl- } \\
\text { prednisolone } \\
0.5 \mathrm{mg} / \mathrm{kg} / \text { day } \\
\text { IV q } 12 \mathrm{~h} \\
\text { for } 7 \text { days } \\
\\
\end{array}$ & 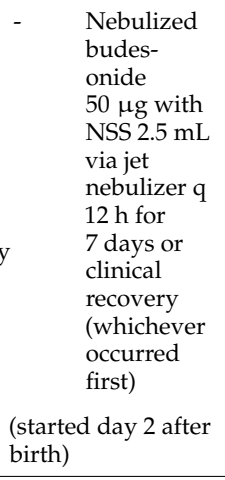 & 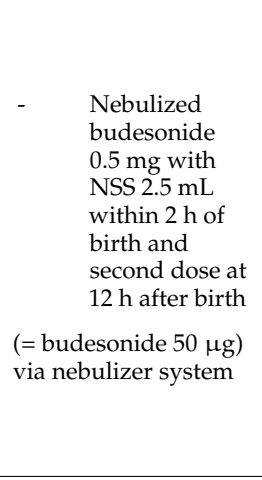 & $\begin{array}{l}\text { - } \quad \text { PS } 100 \\
\quad \mathrm{mg} / \mathrm{kg}\end{array}$ & $\begin{array}{l}\text { Dexamethasone } \\
0.5 \mathrm{mg} / \mathrm{kg} \mathrm{IV} \mathrm{q} \\
12 \mathrm{~h} \text { for days } 1-3, \\
\text { then } 0.25 \mathrm{mg} / \mathrm{kg} \mathrm{q} \\
12 \mathrm{~h} \text { for days } 4-7\end{array}$ & $\begin{array}{l} \\
\text { Dexamethasone } \\
0.25 \mathrm{mg} / \mathrm{kg} \\
\text { IV } 12 \mathrm{~h} \\
\text { for } 3 \text { days } \\
\text { (started } 24-36 \mathrm{~h} \\
\text { after birth) }\end{array}$ & $\begin{array}{l}\text { Methyl- } \\
\text { prednisolone } \\
0.5 \mathrm{mg} / \mathrm{kg} / \\
\text { day q } 12 \mathrm{~h} \\
\text { Nebulized } \\
\text { budes- } \\
\text { onide } \\
\text { respirator } \\
\text { suspen- } \\
\text { sion } \\
0.5 \mathrm{mg}+ \\
\mathrm{NSS} \\
2.5 \mathrm{~mL} \mathrm{q} \\
12 \mathrm{~h} \mathrm{for} \\
7 \text { days }\end{array}$ \\
\hline $\begin{array}{l}\text { Comparator } \\
\text { (2) }\end{array}$ & & & $\begin{array}{l}\text { Nebulized } \\
\text { budesonide } 50 \mu \mathrm{g} \\
+ \text { NSS } 2.5 \mathrm{~mL} \text { via } \\
\text { jet nebulizer q } 12 \mathrm{~h} \\
\text { for } 7 \text { days (started } \\
24-36 \mathrm{~h} \text { after birth) }\end{array}$ & $\begin{array}{ll}\text { - } & \text { Nebulized } \\
\text { budes- } \\
\text { onide } \\
50 \mu \mathrm{g}+ \\
\text { NSS } 2.5 \mathrm{~mL} \\
\text { via jet } \\
\text { nebulizer q } \\
12 \mathrm{~h} \text { for } \\
7 \text { days } \\
5 \% \\
\text { Dextrose } \\
\text { IV }\end{array}$ & - & - & - & - & & - \\
\hline Follow up & - & - & $\begin{array}{l}\text { Weekly for } 2 \\
\text { weeks, } \\
\text { then monthly }\end{array}$ & - & $\begin{array}{l}\text { Once every } 2 \\
\text { weeks for } 3 \\
\text { months }\end{array}$ & - & - & - & $\mathrm{r}$ & $\begin{array}{l}1,3 \text {, and } 6 \\
\text { months for any } \\
\text { complications }\end{array}$ \\
\hline
\end{tabular}


Table 1. Cont

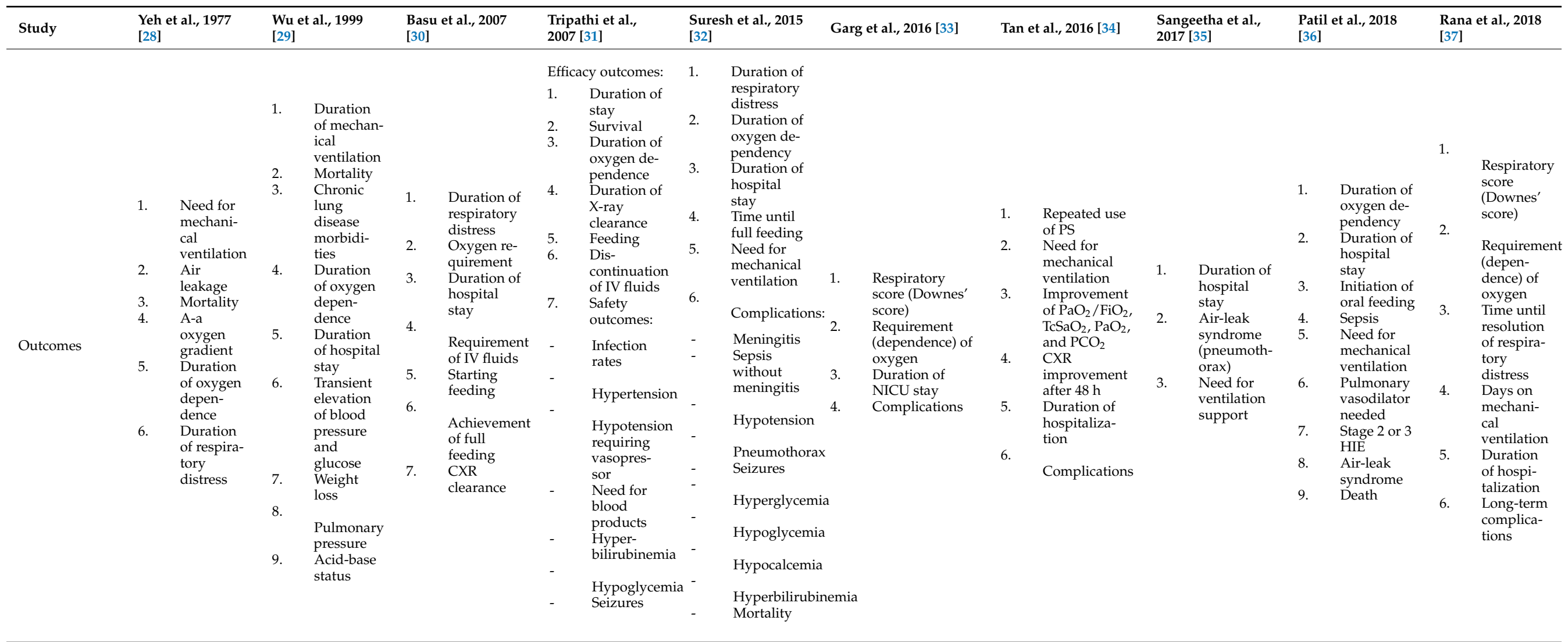

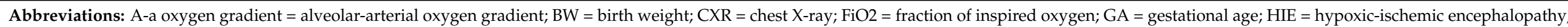

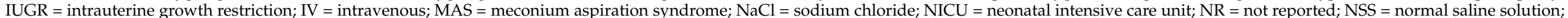

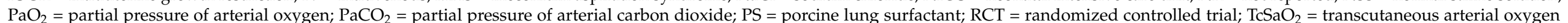
saturation. 


\subsubsection{Downes' Score}

Two RCTs reported Downes' score [33,37]. One study [33] reported a lower mean Downes' score (over 5 days) in the nebulized budesonide group than in the control group $(p<0.05)$. The other study [37] showed a lower median Downes' score (days 2-7) in the nebulized budesonide with IV methylprednisolone group than in the control group $(p<0.05)$.

\subsubsection{Duration of X-ray Clearance}

Three RCTs $[28,30,31]$ provided evidence regarding the duration of X-ray clearance. Two RCTs [30,31] determined this effect of nebulized budesonide and IV methylprednisolone with very low-quality evidence (MD, -5.99 days; $95 \% \mathrm{CI},-12.53$ to 0.56 days and MD, -5.83 days; $95 \% \mathrm{CI},-12.51$ to 0.85 days, respectively) (Figure 2D, Table 2, and Supplementary Tables S3 and S4).

(A)

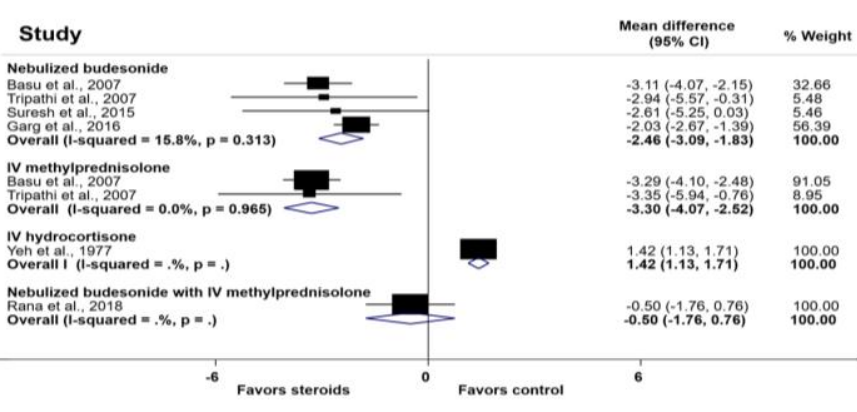

(C)

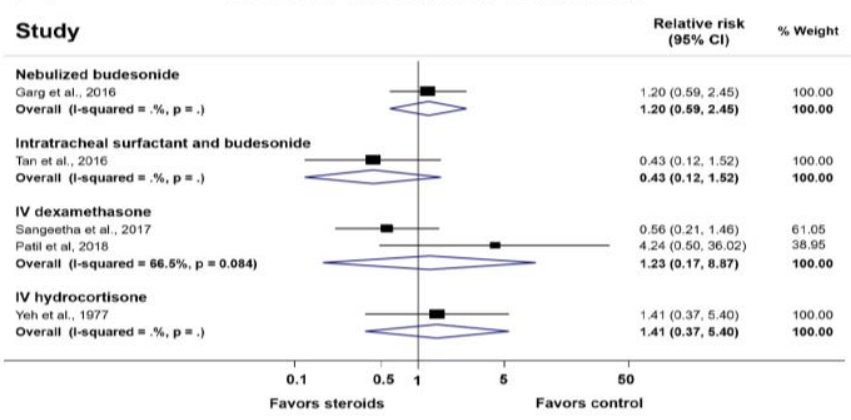

(E) Duration of hospitalization, NICU stay, and PICU stay (days)

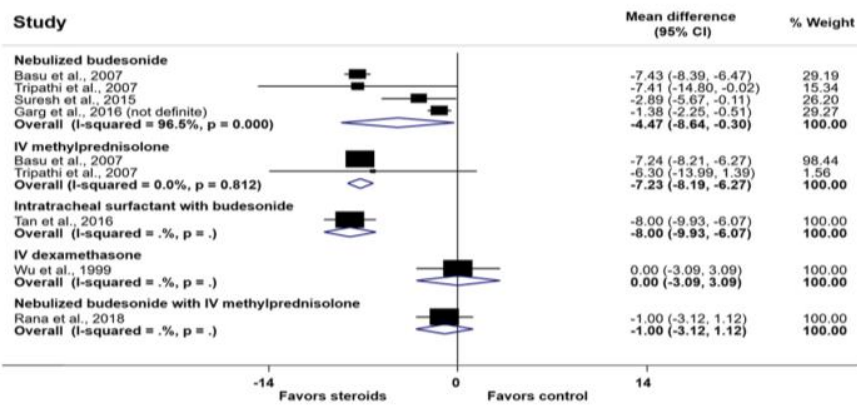

(B)

Duration of oxygen requirement (days)

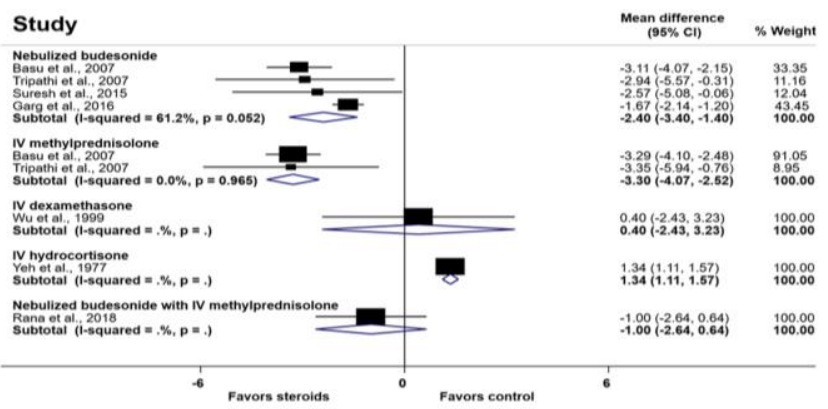

(D)

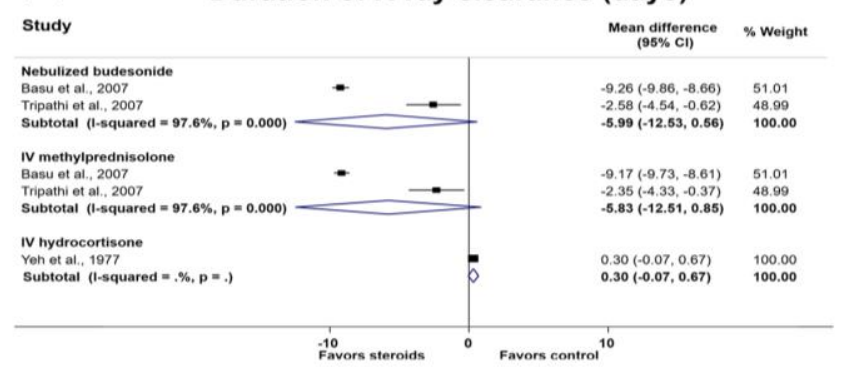

(F)

Time until achievement of full feeding (days)

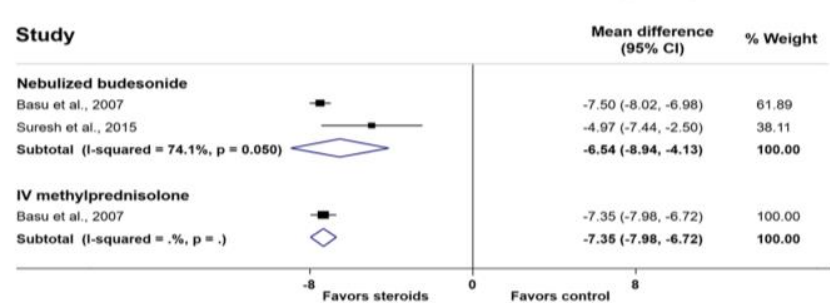

Figure 2. Forest plots of the effects of steroids in infants with meconium aspiration syndrome. (A) Duration of respiratory distress; (B) duration of oxygen requirement; (C) need for mechanical ventilation; (D) duration of X-ray clearance; (E) duration of hospitalization, NICU stay, and PICU stay; (F) time until achievement of full feeding. 
(A)

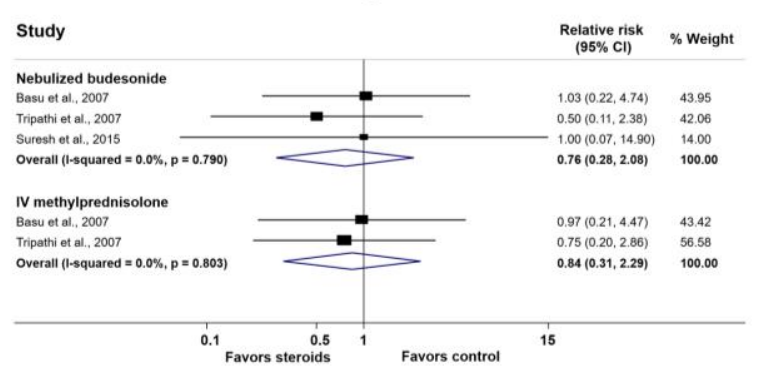

(C)

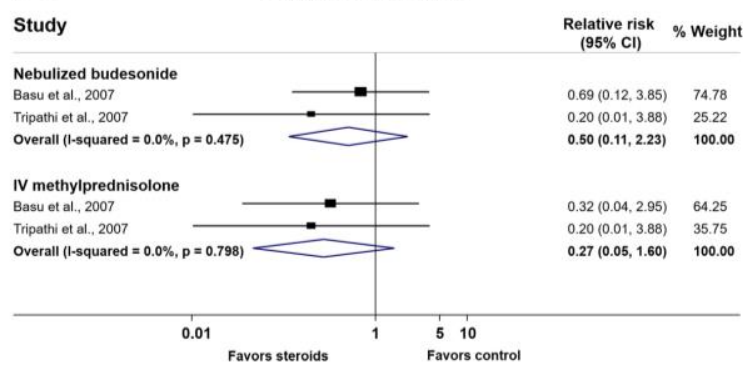

(E)

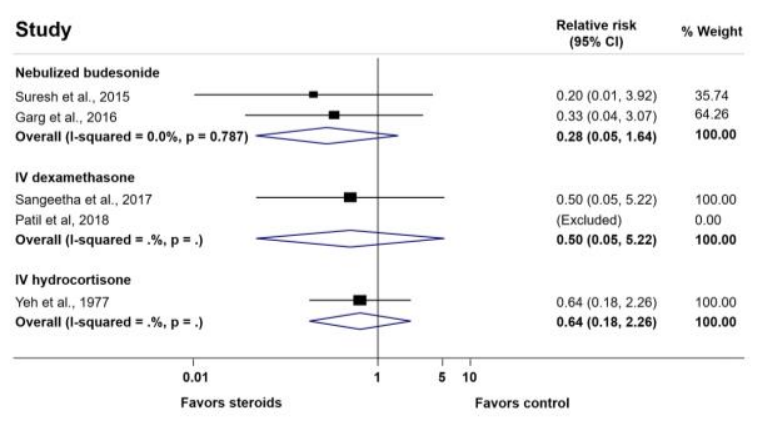

(B)

Sepsis without meningitis

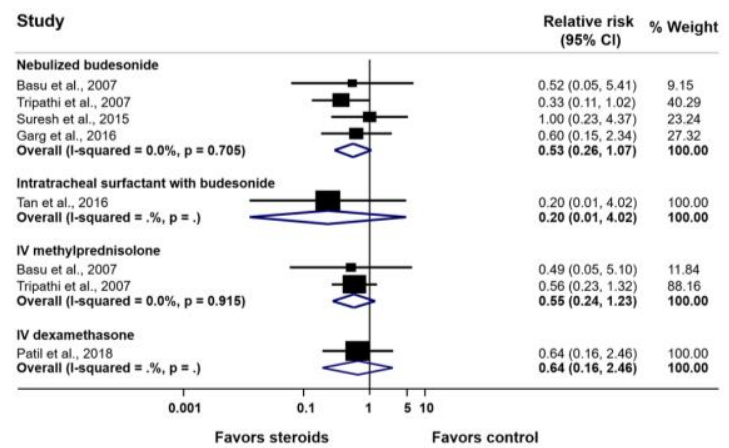

(D)

Oral thrush

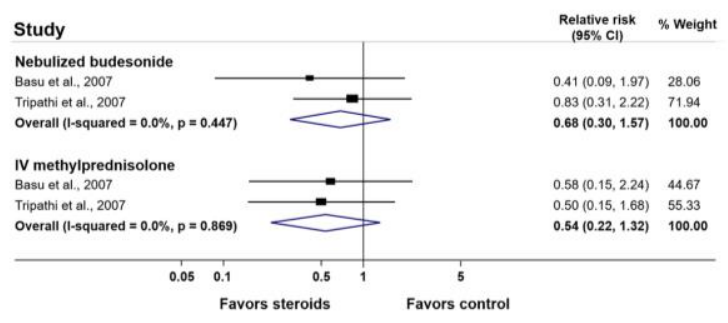

(F)

Death

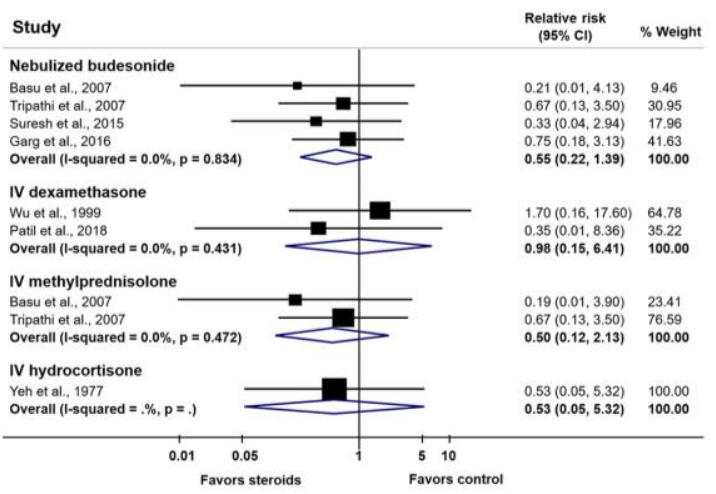

Figure 3. Forest plots of the complications and adverse effects of steroids in infants with meconium aspiration syndrome. (A) Meningitis; (B) sepsis without meningitis; (C) fungal infection; (D) oral thrush; (E) pneumothorax; (F) death.

Table 2. GRADE summary of findings: Effect of steroids on clinical outcomes in neonates with MAS.

Patient or Population: Neonates with MAS

Intervention: Steroids

Comparison: Placebo, no treatment, or usual care

\begin{tabular}{|c|c|c|c|c|c|c|}
\hline \multirow[b]{2}{*}{ Outcomes } & \multirow{2}{*}{$\begin{array}{l}\text { No. of } \\
\text { Participants } \\
\text { (Studies) }\end{array}$} & \multirow{2}{*}{$\begin{array}{l}\text { Relative } \\
\text { Effects } \\
(95 \% \text { CI })\end{array}$} & \multicolumn{2}{|c|}{ Absolute Effect Estimates } & \multirow{2}{*}{$\begin{array}{l}\text { Certainty/Quality } \\
\text { of Evidence }\end{array}$} & \multirow{2}{*}{$\begin{array}{l}\text { Plain Language } \\
\text { Summary }\end{array}$} \\
\hline & & & $\begin{array}{l}\text { Baseline Risk for } \\
\text { Control Group }{ }^{1}\end{array}$ & $\begin{array}{l}\text { Difference } \\
(95 \% \text { CI) }\end{array}$ & & \\
\hline \multicolumn{7}{|c|}{ Duration of respiratory distress (days) } \\
\hline Budesonide & $\begin{array}{l}208 \\
\text { (4 studies) }\end{array}$ & - & $\begin{array}{l}\text { The median } \\
\text { duration of } \\
\text { respiratory } \\
\text { distress in the } \\
\text { control groups } \\
\text { was } 5.71 \text { days }\end{array}$ & $\begin{array}{l}\text { MD }-2.46 \text { days } \\
(-3.09 \text { to } \\
-1.83)\end{array}$ & $\begin{array}{l}\text { Moderate } \\
\oplus \oplus \oplus \ominus \\
\text { (serious risk of } \\
\text { bias) }\end{array}$ & $\begin{array}{l}\text { Budesonide } \\
\text { probably reduces } \\
\text { duration of } \\
\text { respiratory } \\
\text { distress. }\end{array}$ \\
\hline
\end{tabular}


Table 2. Cont.
Patient or Population: Neonates with MAS
Intervention: Steroids
Comparison: Placebo, no treatment, or usual care

\begin{tabular}{|c|c|c|c|c|c|c|}
\hline \multirow{2}{*}{ Outcomes } & \multirow{2}{*}{$\begin{array}{l}\text { No. of } \\
\text { Participants } \\
\text { (Studies) }\end{array}$} & \multirow{2}{*}{$\begin{array}{l}\text { Relative } \\
\text { Effects } \\
(95 \% \text { CI) }\end{array}$} & \multicolumn{2}{|c|}{ Absolute Effect Estimates } & \multirow{2}{*}{$\begin{array}{l}\text { Certainty/Quality } \\
\text { of Evidence }\end{array}$} & \multirow{2}{*}{$\begin{array}{l}\text { Plain Language } \\
\text { Summary }\end{array}$} \\
\hline & & & $\begin{array}{l}\text { Baseline Risk for } \\
\text { Control Group }{ }^{1}\end{array}$ & $\begin{array}{l}\text { Difference } \\
(95 \% \text { CI })\end{array}$ & & \\
\hline $\begin{array}{l}\text { Methyl- } \\
\text { prednisolone }\end{array}$ & $\begin{array}{l}96 \\
\text { (2 studies) }\end{array}$ & - & $\begin{array}{l}\text { The median } \\
\text { duration of } \\
\text { respiratory } \\
\text { distress in the } \\
\text { control groups } \\
\text { was } 5.71 \text { days }\end{array}$ & $\begin{array}{l}\text { MD }-3.30 \text { days } \\
(-4.07 \text { to } \\
-2.52)\end{array}$ & $\begin{array}{l}\text { Moderate } \\
\oplus \oplus \oplus \ominus \\
\text { (serious risk of } \\
\text { bias) }\end{array}$ & $\begin{array}{l}\text { Methylprednisolone } \\
\text { probably reduces } \\
\text { duration of } \\
\text { respiratory } \\
\text { distress. }\end{array}$ \\
\hline
\end{tabular}

\section{Duration of oxygen requirement (days)}

\begin{tabular}{|c|c|c|c|c|c|c|}
\hline Budesonide & $\begin{array}{l}208 \\
\text { (4 studies) }\end{array}$ & - & $\begin{array}{l}\text { The median } \\
\text { duration of } \\
\text { oxygen } \\
\text { requirement in } \\
\text { the control } \\
\text { groups was } 4.94 \\
\text { days }\end{array}$ & $\begin{array}{l}\text { MD }-2.40 \text { days } \\
(-3.40 \text { to } \\
-1.40)\end{array}$ & $\begin{array}{l}\text { Low } \oplus \oplus \ominus \ominus \\
\text { (serious risk of } \\
\text { bias, serious } \\
\text { inconsistency) }\end{array}$ & $\begin{array}{l}\text { Budesonide may } \\
\text { reduce duration of } \\
\text { oxygen } \\
\text { requirement. }\end{array}$ \\
\hline $\begin{array}{l}\text { Methyl- } \\
\text { prednisolone }\end{array}$ & $\begin{array}{l}96 \\
\text { (2 studies) }\end{array}$ & - & $\begin{array}{l}\text { The median } \\
\text { duration of } \\
\text { oxygen } \\
\text { requirement in } \\
\text { the control } \\
\text { groups was } 4.94 \\
\text { days }\end{array}$ & $\begin{array}{l}\text { MD }-3.30 \text { days } \\
(-4.07 \text { to } \\
-2.52)\end{array}$ & $\begin{array}{l}\text { Moderate } \\
\oplus \oplus \oplus \ominus \\
\text { (serious risk of } \\
\text { bias) }\end{array}$ & $\begin{array}{l}\text { Methylprednisolone } \\
\text { probably reduces } \\
\text { duration of } \\
\text { oxygen } \\
\text { requirement. }\end{array}$ \\
\hline
\end{tabular}

\section{Need for mechanical ventilation}

\begin{tabular}{|c|c|c|c|c|}
\hline $\begin{array}{l}\text { Dexa- } \\
\text { methasone }\end{array}$ & $\begin{array}{l}130 \\
\text { (2 studies) }\end{array}$ & $\begin{array}{l}1.23 \\
(0.17 \text { to } 8.87)\end{array}$ & $25.00 \%$ & $\begin{array}{l}5.75(-20.75 \text { to } \\
196.75)\end{array}$ \\
\hline
\end{tabular}

\section{Very low}

$\oplus \ominus \ominus \ominus$

(serious risk of

bias, serious

inconsistency,

and serious

imprecision)
The effect of dexamethasone on the need for mechanical ventilation is very uncertain.

\section{Duration of hospitalization, NICU stay, and PICU stay (days)}

\begin{tabular}{|c|c|c|c|c|c|c|}
\hline Budesonide & $\begin{array}{l}208 \\
\text { (4 studies) }\end{array}$ & - & $\begin{array}{l}\text { The median } \\
\text { duration of hospi- } \\
\text { talization/NICU } \\
\text { stay in the control } \\
\text { groups was } 14 \\
\text { days }\end{array}$ & $\begin{array}{l}\text { MD }-4.47 \text { days } \\
(-8.64 \text { to } \\
-0.30)\end{array}$ & $\begin{array}{l}\text { Low } \oplus \oplus \ominus \ominus \\
\text { (serious risk of } \\
\text { bias, serious } \\
\text { inconsistency) }\end{array}$ & $\begin{array}{l}\text { Budesonide may } \\
\text { reduce the } \\
\text { duration of } \\
\text { hospitalization, } \\
\text { NICU stay, and } \\
\text { PICU stay. }\end{array}$ \\
\hline $\begin{array}{l}\text { Methyl- } \\
\text { prednisolone }\end{array}$ & $\begin{array}{l}96 \\
\text { (2 studies) }\end{array}$ & - & $\begin{array}{l}\text { The median } \\
\text { duration of hospi- } \\
\text { talization/NICU } \\
\text { stay in the control } \\
\text { groups was } 14 \\
\text { days }\end{array}$ & $\begin{array}{l}\text { MD }-7.23 \text { days } \\
(-8.19 \text { to } \\
-6.27)\end{array}$ & $\begin{array}{l}\text { Moderate } \\
\oplus \oplus \oplus \ominus \\
\text { (serious risk of } \\
\text { bias) }\end{array}$ & $\begin{array}{l}\text { Methylprednisolone } \\
\text { probably reduces } \\
\text { the duration of } \\
\text { hospitalization, } \\
\text { NICU stay, and } \\
\text { PICU stay. }\end{array}$ \\
\hline \multicolumn{7}{|c|}{ Infections and death } \\
\hline \multicolumn{7}{|l|}{ Meningitis } \\
\hline Budesonide & $\begin{array}{l}139 \\
\text { (3 studies) }\end{array}$ & $\begin{array}{l}0.76 \\
(0.28 \text { to } 2.08)\end{array}$ & $10.00 \%$ & $\begin{array}{l}-2.40(-7.20 \text { to } \\
10.80)\end{array}$ & $\begin{array}{l}\text { Low } \oplus \oplus \ominus \ominus \\
\text { (serious risk of } \\
\text { bias, serious } \\
\text { imprecision) }\end{array}$ & $\begin{array}{l}\text { Budesonide may } \\
\text { not increase the } \\
\text { number of } \\
\text { participants with } \\
\text { meningitis. }\end{array}$ \\
\hline
\end{tabular}


Table 2. Cont.
Patient or Population: Neonates with MAS
Intervention: Steroids
Comparison: Placebo, no treatment, or usual care

\begin{tabular}{|c|c|c|c|c|c|c|}
\hline \multirow[b]{2}{*}{ Outcomes } & \multirow{2}{*}{$\begin{array}{l}\text { No. of } \\
\text { Participants } \\
\text { (Studies) }\end{array}$} & \multirow{2}{*}{$\begin{array}{l}\text { Relative } \\
\text { Effects } \\
\text { (95\% CI) }\end{array}$} & \multicolumn{2}{|c|}{ Absolute Effect Estimates } & \multirow{2}{*}{$\begin{array}{l}\text { Certainty/Quality } \\
\text { of Evidence }\end{array}$} & \multirow{2}{*}{$\begin{array}{l}\text { Plain Language } \\
\text { Summary }\end{array}$} \\
\hline & & & $\begin{array}{l}\text { Baseline Risk for } \\
\text { Control Group }{ }^{1}\end{array}$ & $\begin{array}{l}\text { Difference } \\
(95 \% \text { CI) }\end{array}$ & & \\
\hline $\begin{array}{l}\text { Methyl- } \\
\text { prednisolone }\end{array}$ & $\begin{array}{l}101 \\
\text { (2 studies) }\end{array}$ & $\begin{array}{l}0.84 \\
(0.31 \text { to } 2.29)\end{array}$ & $10.00 \%$ & $\begin{array}{l}-1.60(-6.90 \text { to } \\
12.90)\end{array}$ & $\begin{array}{l}\text { Low } \oplus \oplus \ominus \ominus \\
\text { (serious risk of } \\
\text { bias, serious } \\
\text { imprecision) }\end{array}$ & $\begin{array}{l}\text { Methylprednisolone } \\
\text { may not increase } \\
\text { the number of } \\
\text { participants with } \\
\text { meningitis. }\end{array}$ \\
\hline
\end{tabular}

\section{Sepsis without meningitis}

\begin{tabular}{|c|c|c|c|c|c|}
\hline Budesonide & $\begin{array}{l}217 \\
\text { (4 studies) }\end{array}$ & $\begin{array}{l}0.53 \\
(0.26 \text { to } 1.07)\end{array}$ & $15.42 \%$ & $\begin{array}{l}-7.25(-11.41 \\
\text { to } 1.08)\end{array}$ & $\begin{array}{l}\text { Low } \oplus \oplus \ominus \ominus \\
\text { (serious risk of } \\
\text { bias, serious } \\
\text { imprecision) }\end{array}$ \\
\hline
\end{tabular}

Budesonide may not increase the number of participants with sepsis without meningitis.

\begin{tabular}{|c|c|c|c|c|c|c|}
\hline $\begin{array}{l}\text { Methyl- } \\
\text { prednisolone }\end{array}$ & $\begin{array}{l}101 \\
\text { (2 studies) }\end{array}$ & $\begin{array}{l}0.55 \\
(0.24 \text { to } 1.23)\end{array}$ & $15.42 \%$ & $\begin{array}{l}-6.94(-11.72 \\
\text { to } 3.55)\end{array}$ & $\begin{array}{l}\text { Low } \oplus \oplus \ominus \ominus \\
\text { (serious risk of } \\
\text { bias, serious } \\
\text { imprecision) }\end{array}$ & $\begin{array}{l}\text { Methylprednisolone } \\
\text { may not increase } \\
\text { the number of } \\
\text { participants with } \\
\text { sepsis without } \\
\text { meningitis. }\end{array}$ \\
\hline \multicolumn{7}{|l|}{ Death } \\
\hline Budesonide & $\begin{array}{l}217 \\
\text { (4 studies) }\end{array}$ & $\begin{array}{l}0.55 \\
(0.22 \text { to } 1.39)\end{array}$ & $11.43 \%$ & $\begin{array}{l}-5.14(-8.92 \text { to } \\
4.46)\end{array}$ & $\begin{array}{l}\text { Low } \oplus \oplus \ominus \ominus \\
\text { (serious risk of } \\
\text { bias, serious } \\
\text { imprecision) }\end{array}$ & $\begin{array}{l}\text { Budesonide may } \\
\text { not increase the } \\
\text { number of } \\
\text { participants with } \\
\text { death. }\end{array}$ \\
\hline $\begin{array}{l}\text { Dexa- } \\
\text { methasone }\end{array}$ & $\begin{array}{l}120 \\
\text { (2 studies) }\end{array}$ & $\begin{array}{l}0.98 \\
(0.15 \text { to } 6.41)\end{array}$ & $11.43 \%$ & $\begin{array}{l}-0.23(-9.72 \text { to } \\
61.84)\end{array}$ & $\begin{array}{l}\text { Low } \oplus \oplus \ominus \ominus \\
\text { (serious risk of } \\
\text { bias, serious } \\
\text { imprecision) }\end{array}$ & $\begin{array}{l}\text { Dexamethasone } \\
\text { may not increase } \\
\text { the number of } \\
\text { participants with } \\
\text { death. }\end{array}$ \\
\hline $\begin{array}{l}\text { Methyl- } \\
\text { prednisolone }\end{array}$ & $\begin{array}{l}101 \\
\text { (2 studies) }\end{array}$ & $\begin{array}{l}0.50 \\
(0.12 \text { to } 2.13)\end{array}$ & $11.43 \%$ & $\begin{array}{l}-5.72(-10.06 \\
\text { to } 12.92)\end{array}$ & $\begin{array}{l}\text { Low } \oplus \oplus \ominus \ominus \\
\text { (serious risk of } \\
\text { bias, serious } \\
\text { imprecision) }\end{array}$ & $\begin{array}{l}\text { Methylprednisolone } \\
\text { may not increase } \\
\text { the number of } \\
\text { participants with } \\
\text { death. }\end{array}$ \\
\hline
\end{tabular}

Abbreviations: $\mathrm{CI}=$ confidence interval; RCTs = randomized controlled trials; MAS = meconium aspiration syndrome; MD = mean difference; NICU = neonatal intensive care unit; PICU = pediatric intensive care unit. Footnote: ${ }^{1}$ Using the median baseline risk in the control group of eligible RCTs. GRADE Working Group grades of evidence. High certainty: We are very confident that the true effect lies close to that of the estimate of the effect. Moderate certainty $\oplus \oplus \oplus \ominus$ : We are moderately confident in the effect estimate: the true effect is likely to be close to the estimate of the effect, but there is a possibility that it is substantially different. Low certainty $\oplus \oplus \ominus \ominus$ : Our confidence in the effect estimate is limited: the true effect may be substantially different from the estimate of the effect. Very low certainty $\ominus \ominus \ominus \ominus$ : We have very little confidence in the effect estimate: the true effect is likely to be substantially different from the estimate of the effect.

\subsubsection{Duration of Hospitalization, Neonatal Intensive Care Unit Stay, and Pediatric Intensive Care Unit Stay}

Nine RCTs [29-37] provided evidence regarding the duration of hospitalization, including intensive care unit (ICU) admission. Four RCTs [30-33] showed that nebulized budesonide may shorten the duration of hospitalization with low-quality evidence (MD, -4.47 days; $95 \%$ CI, -8.64 to -0.30 days). Two RCTs $[30,31]$ showed that IV methylprednisolone probably decreases the length of hospitalization with moderate-quality evidence 
(MD, -7.23 days; $95 \%$ CI, -8.19 to -6.07 days). Three RCTs $[29,35,36]$ provided data on dexamethasone; among these, only one RCT [29] reported the duration of hospitalization (MD, 0.00 days; $95 \%$ CI, -3.09 to 3.09 days) (Figure 2E, Table 2, and Supplementary Tables S3 and S4).

\subsubsection{Time until Achievement of Full Feeding}

Three RCTs [30-32] provided evidence regarding nebulized budesonide on time taken to full feeding in neonates with MAS. Two RCTs $[30,32]$ showed a significantly shorter time until full feeding in the nebulized budesonide group than in the placebo or no treatment group with very low-quality evidence (MD, -6.54 days; $95 \% \mathrm{CI},-8.94$ to -4.13 days) (Figure 2F and Supplementary Tables S3 and S4).

\subsubsection{Duration of IV Fluid Requirement}

One study showed that both nebulized budesonide and IV methylprednisolone reduced the duration of IV fluid requirement in infants with MAS [30] (MD, -6.95 days; $95 \%$ CI, -7.50 to -6.40 days and $\mathrm{MD},-7.06$ days; $95 \% \mathrm{CI},-7.66$ to -6.46 days, respectively) (Supplementary Table S4 and Figure S1).

\subsubsection{Infections and Complications}

We performed meta-analyses of infections and other complications, including pneumothorax, hypotension, hypoglycemia, hyperbilirubinemia, and seizure (Figure 3, Table 2, and Supplementary Tables S3, S4 and Figure S1). There was no significant association of steroids with infections and complications with low- to very low-quality evidence. A metaanalysis could not be performed for hypocalcemia, respiratory arrest, PPHN including the need for pulmonary vasodilators, anemia, stage 2 hypoxic-ischemic encephalopathy, and diarrhea (Supplementary Table S4 and Figure S1). One study [30] revealed no cases of hypertension or hyperglycemia in both the nebulized budesonide and IV methylprednisolone groups. One patient each in the nebulized budesonide and placebo groups developed hyperglycemia in one study [32].

\subsubsection{Death}

Seven RCTs [28-33,36] provided data regarding death. A meta-analysis could be performed for six of these RCTs [29-33,36]. With low-quality evidence, the studies showed that mortality was not reduced with the use of IV dexamethasone [29,36] [RR, $0.98 ; 95 \% \mathrm{CI}, 0.15$ to 6.41; risk difference (RD), -0.23 ; $95 \% \mathrm{CI},-9.72$ to 61.84 ], nebulized budesonide [30-33] (RR, $0.55 ; 95 \% \mathrm{CI}, 0.22$ to $1.39 ; \mathrm{RD},-5.14 ; 95 \% \mathrm{CI},-8.92$ to 4.46$)$, and IV methylprednisolone [30,31] (RR, $0.50 ; 95 \% \mathrm{CI}, 0.12$ to $2.13 ; \mathrm{RD},-5.72 ; 95 \% \mathrm{CI},-10.06$ to 12.92 ).

\section{Discussion}

We included all available RCTs to evaluate the effectiveness, safety, and adverse effects of different types of steroids in infants with MAS. Our results show the benefits of both nebulized budesonide and IV methylprednisolone on the duration of respiratory distress, oxygen requirement, and hospitalization, including ICU admission (moderateto low-quality evidence). Nebulized budesonide shortens the time until achievement of full feeding (very low-quality evidence) without statistically significant increases in the incidence of infections and complications (low- to very low-quality evidence). There was no reduction of mortality regardless of the type of steroid administration.

We performed a rigorous and systematic search to identify relevant studies using the revised version of the Cochrane risk-of-bias tool. We assessed the certainty of evidence for each outcome using the GRADE approach $[16,21]$ our protocol was registered in PROSPERO, and reporting the results followed the PRISMA statement.

Based on the pathophysiology of MAS, treatments to reduce inflammation and cytokine production should benefit patients with MAS. The instillation of budesonide with surfactant has been shown to improve the respiratory status in animal studies [38]. Man- 
agement of MAS in infants mainly involves supportive respiratory and cardiovascular care, with other modalities such as surfactants [39]. Insufficient treatment data in previous studies (e.g., steroids) were investigated in our study.

The effect of steroids on MAS in infants was evaluated in a Cochrane meta-analysis [15]; however, it included only studies by Yeh et al. [28] and Wu et al. [29] This meta-analysis showed no effect of steroids on the duration of oxygen therapy or mortality rate because of insufficient evidence.

Our study updated the current data regarding the effects of steroids on MAS with more relevant clinical outcomes and complications. In addition, other outcomes, such as pulmonary hypertension and respiratory arrest, were additionally identified and are shown in Supplementary Figure S1.

There was no significant difference in the occurrence of PPHN among neonates with MAS with/without steroids [30,33,34,36]. Pneumothorax also showed no difference in neonates with/without budesonide [32,33]. No significant increase in either hypertension or hyperglycemia among neonates with steroids was reported [30,32].

\section{Strengths and Limitations}

Our comprehensive and systematic search with separate and independent screening, searching, study selection, data extraction, quality assessment of this review focused on important outcomes. The GRADE approach was used to rate the quality of evidence, including risk of bias, inconsistency, indirectness, imprecision, and publication bias.

There are several limitations. Even though only RCTs were evaluated, the quality varied from moderate to very low. For outcomes, the quality rating of evidence was decreased by one level based on the risk of bias (Table 2 and Supplementary Table S3). Adequate allocation sequence and concealment were reported in five studies [28,30-33] and one study [30], respectively. A blinding process was performed in three studies (Supplementary Table S2) $[28,29,31]$. For some outcomes, we decreased the quality of evidence rating by one level based on high heterogeneity $\left(\mathrm{I}^{2}>50 \%\right.$ ) (Table 2 and Supplementary Table S3).

Notably, most of the included studies were performed in Asian populations, and 7 of the 10 studies were conducted in India or countries with low resource settings.

Patients were not severe as not much initial requirement of mechanical ventilation in most studies, and data on severity of pulmonary disease, such as oxygen index, were not provided. The analysis is also limited by the different methodology performed in each study and the relatively small number of infants available to assess each outcome. There is variability between the reported studies. Otherwise, the long-term outcomes of steroids, such as neurodevelopmental results, should be followed. Thus, the largesample, uniform methodology and high-quality RCTs involving different populations should further confirm the effect of steroids in infants with MAS.

\section{Conclusions}

For infants with MAS, certain types of steroids may be beneficial in reducing the duration of respiratory distress, oxygen requirement, hospital stay, and time until achievement of full feeding without short-term complications. However, no benefits of decreased mortality in any types of steroid use.

Supplementary Materials: The following are available online at https:/ / www.mdpi.com/article/10 $.3390 /$ medicina57111281/s1. Supplement Table S1: baseline maternal and neonatal characteristics of the included studies; Supplement Table S2: risk-of-bias summary of the included studies using revised Cochrane risk-of-bias tool for randomized trials; Supplement Table S3: GRADE evidence profile of the evidence outcomes; Supplement Table S4: summary results of the included studies categorized by outcomes; Supplement Figure S1: results of the outcomes in the systematic review and meta-analysis. 
Author Contributions: All authors had full access to all of the data in the study and take responsibility for the integrity of the data and the accuracy of the data analysis. Study concept and design: N.P. and W.T.; acquisition, analysis, and interpretation of data: N.P. and W.T.; drafting of the manuscript: N.P., T.U., and W.T.; critical revision of the manuscript for important intellectual content: N.P., T.U. and W.T.; statistical analysis: W.T.; administrative, technical, or material support: Not applicable. All authors have read and agreed to the published version of the manuscript.

Funding: This research received no external funding.

Institutional Review Board Statement: Not applicable.

Informed Consent Statement: Not applicable.

Data Availability Statement: The data supporting the findings of this study are available within the article.

Acknowledgments: We wish to acknowledge and thank Gordon H. Guyatt for all suggestions.

Conflicts of Interest: The authors declare no conflict of interest.

\section{References}

1. Raju, U.; Sondhi, V.; Patnaik, S.K. Meconium Aspiration Syndrome: An Insight. Med. J. Armed Forces India 2010, 66, 152-157. [CrossRef]

2. Parker, T.A.; Kinsella, J.P. Respiratory Disorders in the Term Infant. In Avery's Diseases of the Newborn, 10th ed.; Gleason, C.A., Juul, S.E., Eds.; Elsevier: Philadelphia, PA, USA, 2018.

3. Gelfand, S.L.; Fanaroff, J.M.; Walsh, M.C. Meconium stained fluid: Approach to the mother and the baby. Pediatr. Clin. N. Am. 2004, 51, 655-667. [CrossRef]

4. Greenough, A.; Pulikot, A.; Dimitriou, G. Prevention and management of meconium aspiration syndrome-assessment of evidence based practice. Eur. J. Pediatr. 2005, 164, 329-330. [CrossRef]

5. Sawyer, T.L.; Rosenkrantz, T. Intubation and Tracheal Suctioning for Meconium Aspiration. Available online: https://emedicine. medscape.com/article/1413467 (accessed on 19 November 2020).

6. Viraraghavan, V.R.; Nangia, S.; Prathik, B.H.; Madarkar, B.S.; Rani, D.; Saili, A. Yield of meconium in non-vigorous neonates undergoing endotracheal suctioning and profile of all neonates born through meconium-stained amniotic fluid: A prospective observational study. Paediatr. Int. Child Health 2018, 38, 266-270. [CrossRef]

7. Vivian-Taylor, J.; Sheng, J.; Hadfield, R.M.; Morris, J.M.; Bowen, J.R.; Roberts, C.L. Trends in obstetric practices and meconium aspiration syndrome: A population-based study. BJOG 2011, 118, 1601-1607. [CrossRef]

8. Paudel, P.; Sunny, A.K.; Poudel, P.G.; Gurung, R.; Gurung, A.; Bastola, R.; Chaudhary, R.N.; Budhathoki, S.S.; Ashish, K.C. Meconium aspiration syndrome: Incidence, associated risk factors and outcome-evidence from a multicentric study in lowresource settings in Nepal. J. Paediatr. Child Health 2020, 56, 630-635. [CrossRef] [PubMed]

9. Singh, B.S.; Clark, R.H.; Powers, R.J.; Spitzer, A.R. Meconium aspiration syndrome remains a significant problem in the NICU: Outcomes and treatment patterns in term neonates admitted for intensive care during a ten-year period. J. Perinatol. 2009, 29, 497-503. [CrossRef]

10. Gurubacharya, S.M.; Rajbhandari, S.; Gurung, R.; Rai, A.; Mishra, M.; Sharma, K.; Aryal, D. Risk factors and outcome of neonates born through meconium stained amniotic fluid in a tertiary hospital of Nepal. J. Nepal Paediatr. Soc. 2015, 35, 44-48. [CrossRef]

11. Sharma, V.; Berkelhamer, S.; Lakshminrusimha, S. Persistent pulmonary hypertension of the newborn. Matern. Health Neonatol. Perinatol. 2015, 1, 14. [CrossRef] [PubMed]

12. Hidalgo, H.A.; Helmke, R.J.; German, V.F.; Mangos, J.A. The effects of cyclosporine and dexamethasone on an alveolar macrophage cell line (NR8383). Transplantation 1992, 53, 620-623. [CrossRef]

13. Barnes, P.J.; Pedersen, S. Efficacy and safety of inhaled corticosteroids in asthma. Report of a workshop held in Eze, France, October 1992. Am. Rev. Respir. Dis. 1993, 148 Pt 2, S1-S26. [CrossRef] [PubMed]

14. Brattsand, R.; Linden, M. Cytokine modulation by glucocorticoids: Mechanisms and actions in cellular studies. Aliment. Pharmacol. Ther. 1996, 10 (Suppl. S2), 81-90. [CrossRef]

15. Ward, M.; Sinn, J. Steroid therapy for meconium aspiration syndrome in newborn infants. Cochrane Database Syst. Rev. 2003, 2003, Cd003485. [CrossRef]

16. Contributed by the CLARITY Group at McMaster University Tool to Assess Risk of Bias in Randomized Controlled Trials. Available online: https://www.evidencepartners.com/resources/methodological-resources/tool-to-assess-risk-of-biasin-randomized-controlled-trials-distillersr (accessed on 24 February 2021).

17. Higgins, J.P.T.; Thomas, J.; Chandler, J.; Cumpston, M.; Li, T.; Page, M.J.; Welch, V.A. (Eds.) Cochrane Handbook for Systematic Reviews of Interventions, 2nd ed.; John Wiley \& Sons: Chichester, UK, 2019.

18. Higgins, J.P.; Thompson, S.G. Quantifying heterogeneity in a meta-analysis. Stat. Med. 2002, 21, 1539-1558. [CrossRef] [PubMed]

19. Higgins, J.P.; Thompson, S.G.; Deeks, J.J.; Altman, D.G. Measuring inconsistency in meta-analyses. BMJ (Clin. Res. Ed.) 2003, 327, 557-560. [CrossRef] 
20. DerSimonian, R.; Laird, N. Meta-analysis in clinical trials. Control Clin. Trials 1986, 7, 177-188. [CrossRef]

21. Guyatt, G.H.; Oxman, A.D.; Vist, G.E.; Kunz, R.; Falck-Ytter, Y.; Alonso-Coello, P.; Schünemann, H.J. GRADE: An emerging consensus on rating quality of evidence and strength of recommendations. BMJ (Clin. Res. Ed.) 2008, 336, 924-926. [CrossRef]

22. Guyatt, G.H.; Oxman, A.D.; Vist, G.; Kunz, R.; Brozek, J.; Alonso-Coello, P.; Montori, V.; Akl, E.A.; Djulbegovic, B.; Falck-Ytter, Y.; et al. GRADE guidelines: 4. Rating the quality of evidence-Study limitations (risk of bias). J. Clin. Epidemiol. 2011, 64, 407-415. [CrossRef]

23. Guyatt, G.H.; Oxman, A.D.; Kunz, R.; Brozek, J.; Alonso-Coello, P.; Rind, D.; Devereaux, P.J.; Montori, V.M.; Freyschuss, B.; Vist, G.; et al. GRADE guidelines 6. Rating the quality of evidence-Imprecision. J. Clin. Epidemiol. 2011, 64, 1283-1293. [CrossRef]

24. Guyatt, G.H.; Oxman, A.D.; Kunz, R.; Woodcock, J.; Brozek, J.; Helfand, M.; Alonso-Coello, P.; Glasziou, P.; Jaeschke, R.; Akl, E.A.; et al. GRADE guidelines: 7. Rating the quality of evidence-Inconsistency. J. Clin. Epidemiol. 2011, 64, 1294-1302. [CrossRef]

25. Guyatt, G.H.; Oxman, A.D.; Kunz, R.; Woodcock, J.; Brozek, J.; Helfand, M.; Alonso-Coello, P.; Falck-Ytter, Y.; Jaeschke, R.; Vist, G.; et al. GRADE guidelines: 8. Rating the quality of evidence-Indirectness. J. Clin. Epidemiol. 2011, 64, 1303-1310. [CrossRef]

26. Guyatt, G.H.; Oxman, A.D.; Montori, V.; Vist, G.; Kunz, R.; Brozek, J.; Alonso-Coello, P.; Djulbegovic, B.; Atkins, D.; Falck-Ytter, Y.; et al. GRADE guidelines: 5. Rating the quality of evidence-Publication bias. J. Clin. Epidemiol. 2011, 64, 1277-1282. [CrossRef] [PubMed]

27. Davey, A.M.; Kueser, T.J.; Turner, H.F. Randomized controlled trial of early dexamethasone therapy in the treatment of meconium aspiration syndrome. Pediatr. Res. 1995, 37, 329.

28. Yeh, T.; Srinivasan, G.; Harris, V.; Pildes, R.S. Hydrocortisone therapy in meconium aspiration syndrome: A controlled study. J. Pediatr. 1977, 90, 140-143. [CrossRef]

29. Wu, J.M.; Yeh, T.F.; Wang, J.Y.; Wang, J.N.; Lin, Y.J.; Hsieh, W.S.; Lin, C.H. The role of pulmonary inflammation in the development of pulmonary hypertension in newborn with meconium aspiration syndrome (MAS). Pediatri. Pulm. Suppl. 1999, 18, 205-208. [CrossRef]

30. Basu, S.; Kumar, A.; Bhatia, B.D.; Satya, K.; Singh, T.B. Role of steroids on the clinical course and outcome of meconium aspiration syndrome -A randomized controlled trial. J. Trop. Pediatr. 2007, 53, 331-337. [CrossRef]

31. Tripathi, S.; Saili, A. The effect of steroids on the clinical course and outcome of neonates with Meconium Aspiration Syndrome. J. Trop. Pediatr. 2007, 53, 8-12. [CrossRef]

32. Suresh, R.; Sudha, R.; Pinto, N.; Pradeep, N. Effect of nebulized Budesonide in improving the clinical outcome of neonates with meconium aspiration syndrome. Int. J. Biol. Med. Res. 2015, 6, 4942-4945.

33. Garg, N.; Choudhary, M.; Sharma, D.; Dabi, D.; Choudhary, J.S.; Choudhary, S.K. The role of early inhaled budesonide therapy in meconium aspiration in term newborns: A randomized control study. J. Matern. Fetal Neonatal. Med. 2016, 29, 36-40. [CrossRef]

34. Tan, X.Z.; Wu, S.G.; Zhang, J.H.; Li, X.F.; Gao, P.M.; Wang, Y. Clinical efficacy of porcine pulmonary surfactant combined with budesonide suspension intratracheal instillation in the treatment of neonatal meconium aspiration syndrome. Zhongguo Dang Dai Er Ke Za Zhi 2016, 18, 1237-1241.

35. Sangeetha, T.; Ramanathan, R.; Yogavalli, S. Effectiveness of steroid therapy in newborns with meconium aspiration syndrome. J. Med. Sci. Clin. Res. 2017, 5, 22587-22590.

36. Patil, M.M.; Lakhkar, B.B.; Patil, S.V. Dexamethasone and outcome of meconium aspiration syndrome: Vijayapur, Karnataka experience. Sri Lanka J. Child Health 2018, 47, 21-26. [CrossRef]

37. Rana, K.S.; Konar, M.C.; Islam, K.; Barik, K.L.; Nayek, K.; Datta, A.K. Study on effects of steroid on clinical course, short-term and long-term outcomes in neonates with meconium aspiration syndrome. J. Neonat. Nurs. 2018, 24, 257-260. [CrossRef]

38. Mikolka, P.; Kopincova, J.; Tomcikova Mikusiakova, L.; Kosutova, P.; Antosova, M.; Calkovska, A.; Mokra, D. Effects of surfactant/budesonide therapy on oxidative modifications in the lung in experimental meconium-induced lung injury. J. Physiol. Pharmacol. 2016, 67, 57-65.

39. Monfredini, C.; Cavallin, F.; Villani, P.E.; Paterlini, G.; Allais, B.; Trevisanuto, D. Meconium Aspiration Syndrome: A Narrative Review. Children 2021, 8, 230. [CrossRef] [PubMed] 